\title{
Targeting biofilm inhibition using Quercetin - Interaction with bacterial cell membrane and ROS mediated biofilm control
}

\author{
Sarangapani Sreelatha ${ }^{1,2 *}$ and Ayyavoo Jayachitra ${ }^{3}$
}

${ }^{1}$ Biosym, Singapore MIT Alliance for Research and Technology, Singapore; ${ }^{2}$ Department of Chemistry and Biosciences, SASTRA University, Tamilnadu, India; ${ }^{3}$ School of Biotechnology, Madurai Kamaraj University, Madurai city, India

Corresponding author: Dr. Sarangapani Sreelatha, Biosym, Singapore MIT Alliance for Research \&Technology, Singapore

Submission Date: March 21 ${ }^{\text {st }}$, 2018, Acceptance Date: June $28^{\text {th }}, 2018$, Publication Date: June $30^{\text {th }}, 2018$

Citation: Sreelatha S., Jayachitra A., Targeting biofilm inhibition using Quercetin - Interaction with bacterial cell membrane and ROS mediated biofilm control. Functional Foods in Health and Disease 2018; 8(6): 292-306. DOI: https://doi.org/10.31989/ffhd.v8i6.510

\begin{abstract}
Background: Quercetin is an active nutraceutical ingredient widely distributed in foods, vegetables, fruits, and more. Quercetin is a versatile functional food with extensive protective effects against many infectious and degenerative diseases due to their antioxidant activities. Apsergillus niger is a filamentous fungus and the most abundant mold found in the environment. This fungus has been the source of several bioactive compounds and industrial enzymes through biotransformation.
\end{abstract}

Aim: In this report we emphasized the potential of Aspergillus species for the selective conversion of rutin to quercetin, which involved stereoselective and regiospecific reactions with enhanced production and minimization of the formation of toxic wastes. This fungal microbe was able to transform the complex structure of rutin to quercetin with remarkable catalytic activity for the reaction with high product yield. The quercetin produced demonstrated the ability to inhibit biofilm formation and eradicate established biofilm involving the production of reactive oxygen species (ROS) indicative of membrane activity. These results suggest quercetin may have implications in biofilm control targeting reactive oxygen species as a novel therapeutic strategy.

Methods: Quercetin was synthesized by microbial biotransformation recruiting Aspergillus niger. The synthesis of quercetin was compared with the chemical process. Furthermore, the quercetin produced by the biotransformation process was characterized by high performance thin layer liquid 
chromatography. The quercetin produced was assessed for biological activities. The antimicrobial activity, hemolytic activity, inhibition of biofilm by crystal violet staining, and cell viability by confocal laser scanning microscope was assessed. The membrane interaction effect and oxidant scavenging effect by DPPH, Intracellular ROS release, and lipid peroxidation was measured.

Results: Quercetin produced by microbial transformation demonstrated antimicrobial activity against $S$. aureus by effectively inhibiting the growth and dispersion of preformed biofilms. Quercetin demonstrated a significant free radical scavenging activity and significant inhibition of lipid peroxidation. Significant release of reactive oxygen species was observed in bacterial cells.

Conclusion: In conclusion, the bio transformed quercetin exhibited disruptive potential of biofilm formation by preventing cell surface attachment and biofilm growth. Therefore, it can be suggested that the major public health benefits could be achieved by substantially increasing the consumption of quercetin rich foods.

\section{INTRODUCTION}

Flavonoids are hydroxylated phenolic substances which have gained significant attention in human health as functional foods and novel therapeutics. They are the most widely recognized groups of natural products largely represented in the human diet, being present in plants, seeds, and food stuffs. Many flavonoids found in nature exist as hydroxylated conjugates which contain diverse physiological activities. Flavonoids are known for being strong antioxidants. Significantly, several investigations have been conducted to elucidate the role of flavonoids and their metabolites in their biological actions [1]. These studies promote screening various flavonoids in order to determine the beneficial use of human kind. Epidemiological evidence suggests that flavonoids, i.e. quercetin, keampferol, and more can reduce the incidence of chronic diseases such as cardiovascular diseases, diabetes, atherosclerosis, stroke, and microbial infections [2].

Bacteria living in biofilms establish an active mechanism to leave biofilms and return to the planktonic (free-living) state and are highly resistant to conventional antibiotics and host defences. The main factor contributing to microbial resistance is the biofilm which grows slowly and adopts a phenotype which confers an intrinsic resistance to many antibiotics classes [3], including the $\beta$ lactams. Moreover, $80 \%$ of chronic bacterial infections are biofilm associated. Therefore, the eradication of microorganisms which live as biofilms becomes difficult, as it is the source of many recalcitrant infections. In recent years, significant research has been focused on novel strategies and technological advances accelerating the discovery and development of innovative pharmaceuticals with improved therapeutic activity.

The flavonoid quercetin has gained widespread interest as an alternative medicine to treat the infections caused by drug resistant organisms. Quercetin is abundant in ethnic plants and has a wide range of applications such as antioxidant, free radical scavenger, anticancer, and neuroprotective effects. Quercetin has a long history of use in folk medicine for the treatment of various infectious diseases and their activities are structure dependent [4]. 
The majority of flavonoids are extracted from plant materials. The low extraction yield of phenolic compounds from plants and natural products by physical, physicochemical, and chemical techniques has led to the demand for microbial fermentation techniques that can increase yield and establish an inexhaustible, renewable resource of high-value bioactive products.

Recently, microbial biotransformation has emerged as an alternative strategy. Microbial biotransformation transforms microorganisms in real microbial cell factories to carry out reactions in asymmetric synthesis for the sustainable production of flavonoids. It is a powerful approach where the structures of bioactive natural and synthetic flavonoids can be modified with the advantage of large scale production, which improves the selectivity of the natural products. The prospect of microbial biotransformation for the development and production of flavonoids has increased the demand for novel drugs, as the treatment of various pathological conditions is a cost effective tool which protects the environment by reducing toxic wastes [5]. In this study, we enhanced the production of quercetin from rutin by using Aspergillus niger fungal strain. The production of quercetin from rutin by microbial transformation was compared with the chemical process. Furthermore, we reported the biological activities of bio transformed quercetin against bacterial cells and membrane active potential. Membrane permeabilization and oxidant scavenging effects provide evidence that quercetin produced from the microbial transformation process has antimicrobial properties, such as damaging membrane integrity and performing scavenging activity.

\section{MATERIALS AND METHODS}

\section{Bacterial strain and growth condition}

The chemicals, standard antibiotics and rutin used in this work were purchased from SigmaAldrich Co (MO, USA). The reference microbial culture was obtained from American Type Culture Collection (ATCC), USA. Culture (S. aureus) was inoculated from fresh slopes and incubated with shaking at $37^{\circ} \mathrm{C}$ for 24 hours. Cells were collected by centrifugation and the collected cell debris was washed twice in PBS and suspended to OD $_{520}$ prior to use in biofilm experiments.

\section{Quercetin synthesized from rutin}

The microorganism Aspergillus niger (isolated from soil) was cultured in a potato dextrose agar medium under aerobic conditions. The biotransformation process rutin [6] was solubilized in dimethyl formamide, incubated with the fungal suspension culture at $27^{\circ} \mathrm{C}$, and shaken at $200 \mathrm{rpm}$ for seven days. Control incubation in the sterile liquid medium without the microorganism was systematically included. Flask contents and the suspension culture were separated by filtration. The organic extract was dried on magnesium sulphate, under reduced pressure to yield the crude extract. This crude extract was deposited on silica gel column and eluted with ethyl acetate and methanol mixture. The collected fraction was concentrated to give the metabolite. The final fraction was then purified and characterized using HPTLC where the densitogram quantified the presence of quercetin. 
Antibacterial efficacy

Antimicrobial activity was carried out using the disc diffusion method [7]. The test cultures were swabbed on the top of the solidified medium and allowed to dry for $10 \mathrm{~min}$. Different concentrations of quercetin were used for the test. The loaded discs were placed on the surface of the medium and left for $30 \mathrm{~min}$ at room temperature for the diffusion of the compound. The negative control was also conducted with the solvent. The plates were then incubated at $37^{\circ} \mathrm{C}$ for $24 \mathrm{hrs}$. Then the inhibition zones were recorded and the antibacterial activity was determined by measuring the diameter of the zone of inhibition around the well using the metric scale. Antibacterial activity was expressed as the ratio of the inhibition zone $(\mathrm{mm})$ produced by quercetin.

\section{Biofilm prevention potential: Disruption of preformed Biofilm}

Experiments were conducted based on the methods described previously [8]. Biofilms were allowed to form prior to the addition of quercetin fraction at a final concentration of $200 \mathrm{ug} / \mathrm{ml}$. The formation was achieved by aliquoting $200 \mathrm{ul}\left(1 \times 10^{7} \mathrm{CFU} / \mathrm{ml}\right)$ of the culture into the 96-well plate. The plates were incubated at $37^{\circ} \mathrm{C}$ for $8 \mathrm{~h}$ to allow cell attachment along with duplicate plates. Following incubation different concentration of the quercetin fraction was added and incubated at $37^{\circ} \mathrm{C}$ for $2 \mathrm{~h}$ along with the positive controls respectively. After incubation, the wells were washed with physiological buffered saline (PBS) solution and quantification of biofilm production was established by crystal violet staining method. Experiments were done in triplicates and the reaction mixture was read spectrophotometrically at $570 \mathrm{~nm}$. Inhibition mediated reduction of biofilm formation was calculated by the following formula:

Percentage of Inhibition $=\quad \mathrm{OD}_{\text {control }}-\mathrm{OD}_{\text {Experimental }}$

X 100

OD control

\section{Confocal Imaging}

Prevention of biofilm formation was confirmed using a microscopic technique [9]. Briefly, strains were allowed to grow on round glass slides (diameter $1 \mathrm{~cm}$ ) placed in 24-well polystyrene plates supplemented with the quercetin fraction $(200 \mathrm{ug} / \mathrm{ml})$ and incubated for $24 \mathrm{~h}$ at $37^{\circ} \mathrm{C}$ and stained with acridine orange solution (v/v) for $20 \mathrm{~min}$ at room temperature. Stained glass slides were placed with the biofilm pointed upwards and were imaged in confocal microscopy (Olympus) at 40x magnification. The 488- $\mathrm{nm}$ Ar laser and a 500-640 $\mathrm{nm}$ band pass emission filter were used to excite and detect the stained cells. CLSM images were obtained from the 24-h old control and treated biofilms.

\section{Inhibition of biofilm growth and development}

Experiments were conducted based on the methods [10] described previously. Biofilms were allowed to form prior to the addition of the quercetin fraction at a final concentration of $200 \mathrm{ug} / \mathrm{ml}$. The formation was achieved by aliquoting $200 \mathrm{ul}\left(1 \times 10^{5} \mathrm{CFU} / \mathrm{ml}\right)$ of the culture into the 96-well 
plate. The plates were then incubated at $37^{\circ} \mathrm{C}$ for $4 \mathrm{~h}$ to allow cell attachment along with duplicate plates. Following incubation, a different concentration of the quercetin was added and incubated at $37^{\circ} \mathrm{C}$ for 3 days along with the positive control. After incubation, the wells were washed with physiological buffered saline (PBS) solution and quantification of biofilm production was established using the crystal violet staining method. Experiments were done in triplicates and the reaction mixture was read spectrophotometrically at $570 \mathrm{~nm}$. Inhibition mediated reduction of biofilm formation was calculated using the following formula:

$$
\% \text { of inhibition }=\text { OD in control }- \text { OD in treatment } \times 100 / \mathrm{OD} \text { in control }
$$

\section{Membrane interaction effect}

Propidium iodide (PI) was added to the biofilm suspension ( $\left.1 \times 10^{5} \mathrm{CFU} / \mathrm{ml}\right)$ in $5 \mathrm{mM}$ HEPES and $5 \mathrm{mM}$ glucose (pH7.4). The suspension with the dye was then added to the 96-well plate. After four minutes, quercetin fraction was added to the solution to a final concentration with the MIC value. The fluorescence intensity was measured at the excitation wavelength of $535 \mathrm{~nm}$ and emission wavelength of $617 \mathrm{~nm}$. The uptake of PI was detected [11] by the increase in fluorescence for 10 minutes, which corresponds to the permeabilization of the inner cell membrane.

\section{Hemolytic assay}

The separated red blood cells from the whole blood were suspended with the PBS solution. Deionized water was added to $0.2 \mathrm{ml}$ of human red blood suspension and used as the negative control or PBS solution as the positive control. Quercetin fraction at a concentration of $200 \mathrm{ug} / \mathrm{ml}$ was added to the human red blood suspension with the PBS solution and further incubated for $2 \mathrm{~h}$ at $37^{\circ} \mathrm{C}$ and then centrifuged at $10,000 \mathrm{rpm}$ for $3 \mathrm{~min}$. The optical density of the supernatant was measured at $540 \mathrm{~nm}$ using the microplate reader. The percentage of hemolysis [12] was calculated by the following equation.

Hemolysis $(\%)=\operatorname{Abs}_{540}\left[\right.$ sample treated] $-\mathrm{Abs}_{540}[$ negative control]/Abs540 [positive control] Abs540 [negative control] $\times 100$.

\section{DPPH Scavenging activity}

The inhibition percentage of preformed free radicals is typically employed to express the antioxidant activity and to compare the activity of compounds. The ability of the compounds to bleach 1,1-Diphenyl-2-picryl-hydrazyl (DPPH) [13] can be quantified using a spectrophotometric assay. The extent of scavenging causes a proportionate change in absorption at $518 \mathrm{~nm}$. An exact amount of the methanolic solution of DPPH was added with a different concentration of the quercetin fraction and allowed to stand at room temperature for 30 minutes. Methanol served as the blank. After 30 minutes, the absorbance was measured at $518 \mathrm{~nm}$ and converted into percentage radical scavenging activity as the following:

Scavenging activity $(\%)=\mathrm{A}_{518}[\mathrm{Control}]-\mathrm{A}_{518}\left[\right.$ sample treated] $/ \mathrm{A}_{518}[\mathrm{Control}] \times 100$ 


\section{Intracellular ROS measurement}

Intracellular reactive oxygen species (ROS) production [14] was measured in both treated and control cells using dichloro-dihydro-fluorescein diacetate (DCFH-DA). Briefly, 1x10 ${ }^{5} \mathrm{CFU} / \mathrm{ml}$ cells were exposed to different concentration of quercetin fraction and incubated. After incubation, the treated and control cells were resuspended in $0.5 \mathrm{ml} \mathrm{PBS}$ containing DCFH-DA at $37^{\circ} \mathrm{C}$ for 30 min and then incubated with $4 \mathrm{mM} \mathrm{H}_{2} \mathrm{O}_{2}$ at $37^{\circ} \mathrm{C}$ for $30 \mathrm{~min}$. ROS production of the cells were evaluated by a microplate reader (Perkin-Elmer, MA).

\section{Effect of quercetin on the membrane lipids}

In order to understand the molecular nature of the assault and its protection in plasma membrane RBC ghosts [15] were used. The assay medium assay corresponding to $100 \%$ oxidation was prepared by adding other constituents like ferrous sulphate, ascorbate, and tris buffered saline. The experimental medium corresponding to auto-oxidation contained only the RBC ghosts. All the tubes with and without quercetin fraction $(200 \mathrm{ug} / \mathrm{ml})$ were incubated at $37^{\circ} \mathrm{C}$ for 1 hour. Following the incubation period, $70 \%$ alcohol was added to all the tubes to arrest the reaction. Thiobarbituric acid was then added to all the tubes and boiled in a water bath for 20 minutes. After being cooled to room temperature, the tubes were centrifuged to clear the solution and the supernatants were added with $0.5 \mathrm{ml}$ of acetone. The intensity of the pink color produced was measured at $535 \mathrm{~nm}$ in a spectrophotometer.

\section{Data analysis}

All the parameters studied were subjected to statistical treatment using Sigma Stat statistical package (Version 3.1). The data were expressed as mean \pm S.D $(n=6)$ where ' $n$ ' represents the no of samples. One-way ANOVA, followed by post-hoc analysis using Fischer's LSD, was adopted to all the parameters under study to test the level of statistical significance. The difference was considered significant if $\mathrm{p}<0.05$.

\section{RESULTS}

The increasing failure of chemotherapeutics and antibiotic resistance exhibited by pathogenic microbial infectious agents has led to the screening of several bioactive compounds for their potential antimicrobial activity. Biotransformation with microorganisms is an alternative strategy to produce bioactive compounds [16]. Aspergillus species have been widely established as cell factories for the synthesis of enzymes and metabolites. This species is considered an industrial superstar due to the critical role is has in the fermentation process for the production of biofuels, bioactive compounds, and functional foods for human health [17]. In this study, we report the bioconversion of rutin to quercetin by Aspergillus niger. The microorganism Aspergillus niger effectively transformed rutin to quercetin with higher yield. The final fraction obtained was then purified and characterized using HPTLC (Scheme 1). The maximum yield of quercetin was discovered to be $81 \%$, demonstrating the enzymatic hydrolysis of hesperidinase in Aspergillus niger which catalyzes the terminal rutinoside groups from rutin to quercetin. In the chemical 
transformation process, quercetin was produced by the hydrolysis of rutin catalyzed by hydrochloride acid $(0.2 \%)$, with the maximum yield of quercetin being $40 \%$. The collected fraction was concentrated and characterized using standard analytical methods. This production of quercetin from rutin using aspergillus species reveals the prospect of microbial biotransformation as a cost effective and environment friendly tool for the development of bio actives.

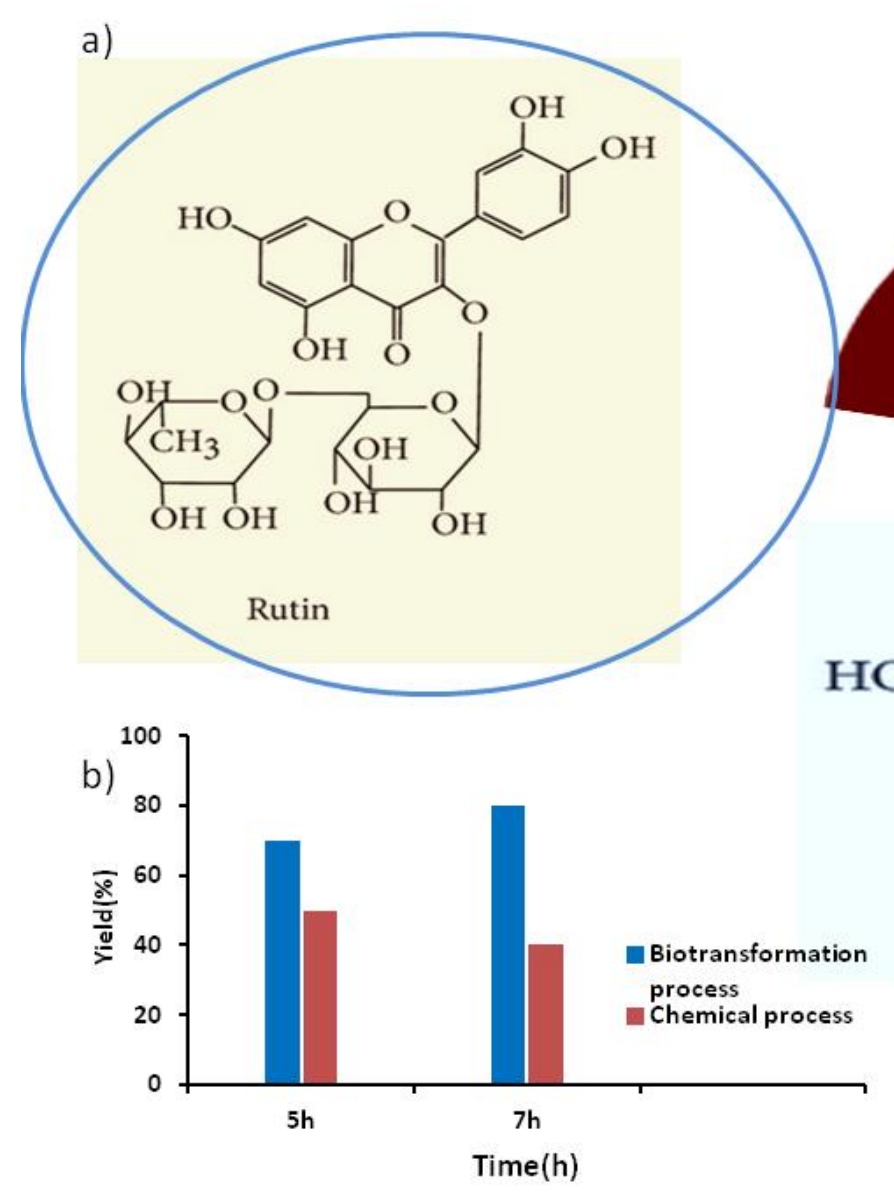

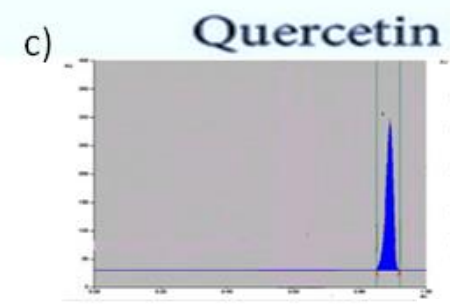

i)

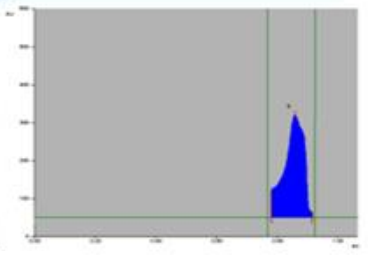

ii)

Figure 1. a) Biotransformation of rutin to quercetin using Aspergillus species b) percentage yield of quercetin from microbial process and chemical process. c) HPTLC densitogram of i) standard quercetin and ii) concentrated metabolite fraction.

The antibacterial potency of quercetin produced was evaluated against various strains like Staphylococcus aureus, Pseudomonas aeruginosa, and Escherichia coli. The minimum inhibitory concentration against various strains increased considerably with increased concentration. However, stronger inhibition was observed in Staphylococcus aureus and the MIC value was 100 $\mathrm{ug} / \mathrm{ml}$. Then the hemolytic activity performed in erythrocytes demonstrated no hemolysis at the MIC values, which was calculated by the $\mathrm{HC}_{50}$ values. In order to determine the ability of quercetin to inhibit biofilm aliquots of $100 \mathrm{ul}$, cell cultures were allowed to grow in a 96 -well plate with 
quercetin (50-200ug/ml) and without quercetin as the control and incubated for $24 \mathrm{hrs}$. After incubation, the wells were rinsed with physiological buffered saline (PBS) solution and quantified as percent biofilms formed. The disruption of preformed biofilm with quercetin $(50-200 \mathrm{ug} / \mathrm{ml})$ was estimated by crystal violet staining method and the images were taken by a digital camera. Compared to control biofilms, a significant reduction in the biofilm formation was observed with quercetin at the concentration of $200 \mathrm{ug} / \mathrm{ml}$. Preformed biofilm of S.aureus were dispersed up to $70 \%$ of $200 \mathrm{ug} / \mathrm{ml}$ of quercetin compared to tetracycline that showed $50 \%$ reduction at the same concentration (Fig-1-a-e).

Flavonoid compounds serve as a promising tool for reducing microbial colonization on surfaces and epithelial mucosa there by inhibiting the cell attachment and subsequently preventing infections. The flavonoid quercetin inhibited cell attachment, which is known to effect the sortase activity [18] and considered to influence the adhesive property of bacterial cell wall there, which suggests the interruption of biofilm development.

a)

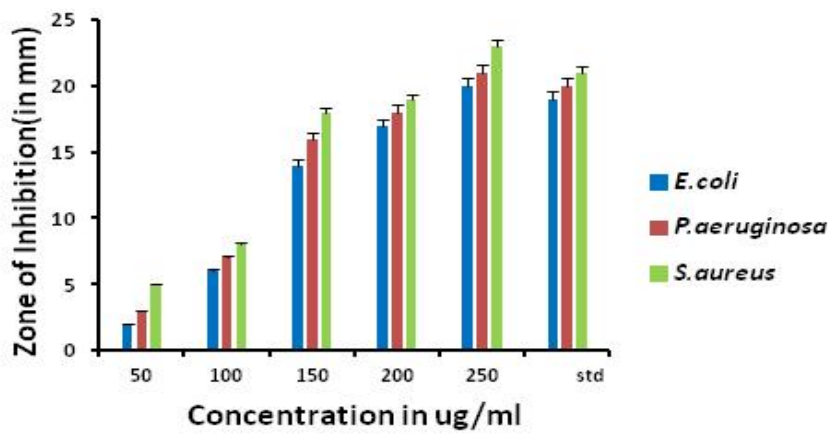

b)

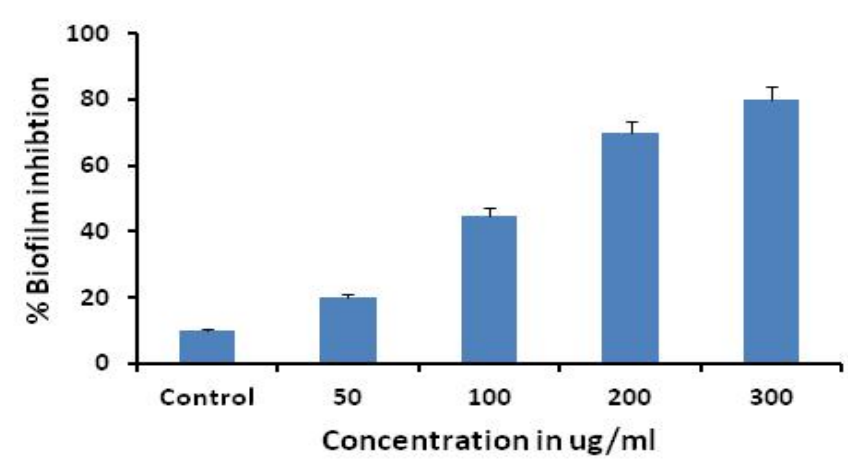

c)

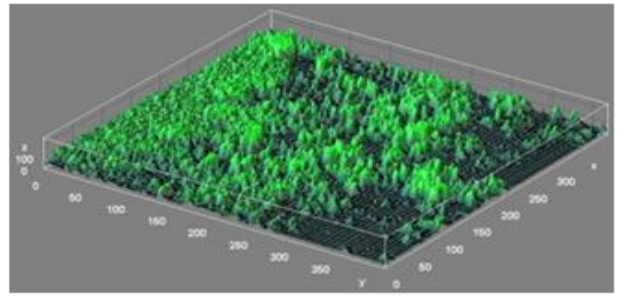

d)

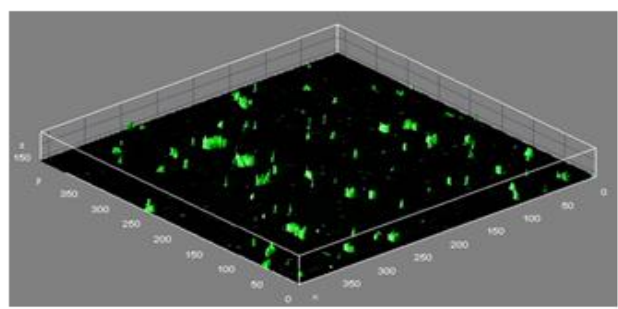

e)

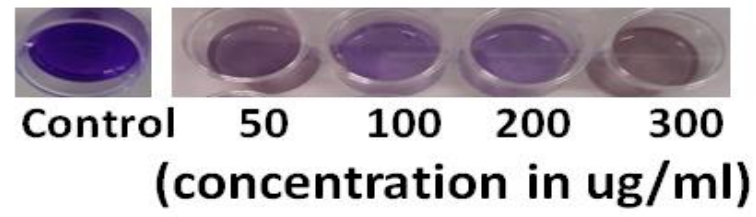

Figure 2. a) Antibacterial activity of bioactive quercetin against Staphylococcus aureus, Escherichia coli, \& Pseudomonas aeruginosa at different concentration. Zone of inhibition in mm. b) Percentage of preformed biofilm bacteria inhibited by bioactive quercetin at a different concentration. c) \& d) Confocal images of the control biofilms with higher surface area and the treated biofilms supplemented with quercetin showed reduction in surface area. The scale bar of the image is $40 \mathrm{um}$. 
In order to determine the viability of biofilms the culture of S.aureus was incubated with quercetin at their respective concentration of $200 \mathrm{ug} / \mathrm{ml}$ or the antibiotics. Biofilms were allowed to form on the pre-sterilized glass surface submerged in the growth media in a petri dish for $24 \mathrm{hr}$ on a rocker. After the incubation period, the slides were removed and rinsed with sterile phosphate buffered saline. Then, the planktonic cells were removed and the biofilms were stained with acridine orange. The cell viability was assessed by a confocal laser scanning microscope (Olympus). Biofilm growth was significantly inhibited after treatment with quercetin (92\%). Tetracycline was less effective in inhibiting the biofilms (78\%) compared to quercetin. Consequently, the antimicrobial action of quercetin may be related to their ability to inactivate microbial adhesion and formation. The key factor in inhibiting the viability is due to surface conditioning by quercetin which prevents the adsorption of substances that include nutrients for cell adhesion by creating an unfavorable environment and simultaneously reducing surface adhesion [19].

The membrane interaction effect of quercetin was evaluated in order to check the potency of quercetin in bacterial cell survival. It was determined using propidium iodide (PI) uptake. The uptake of PI was detected by the increase in fluorescence that correlates to permeabilization. Quercetin was more effective and rapid at permeabilizing the inner membrane at the molar concentration greater than the MIC. These results suggested that membrane permeation may play a key role in the antimicrobial activity, resulting in bacterial toxicity. Therefore, our results revealed that bioactive quercetin kills the bacteria in suspension and prevents biofilm formation. As a result, the antibacterial action of quercetin further demonstrates the membrane interference by reducing the membrane fluidity of the bacterial cells [20].

Quercetin was tested for the scavenging activity against the stable free radical DPPH $(2,2-$ diphenyl-1-picryl hydrazyl). The ability of quercetin to scavenge DPPH was quantified using a spectrophotometric assay. There was a stronger scavenging activity of DPPH observed with quercetin in a dose dependent manner, which confirmed the antioxidant nature of quercetin. These results further support that the functional hydroxyl groups in quercetin mediate their antioxidant effects by scavenging free radicals and confirm that biological activities are structure dependent [21].

Any oxidant entering the biological system can maximally manifest its damaging effects only after entering into the cellular environment and by-passing the endogenous antioxidant system [22]. For entry into the cell or its organelles, an oxidant needs to cross the membrane barriers. Accordingly, in the present study the source of membrane lipids was challenged with an oxidant assault and we studied the effect of quercetin regarding the damage inflicted to the lipid preparation. Lipid peroxidation (LPO), which is the ultimate oxidative damage inflicted to lipids, is one of the best known manifestations of oxidant-induced cell injury [23]. LPO was induced in the plasma membrane preparation using $\mathrm{RBC}$ ghosts, which was followed by measuring the TBARS formed. Quercetin caused a very significant inhibition of the extent of LPO in the lipid preparation, evinced the maximum protection to the plasma membrane, and was very effective in 
counteracting the oxidative damage to cellular macromolecular targets. Altogether, these results demonstrate how quercetin is non-toxic and does not initiate the process of oxidation in the normal cellular membrane.

a)

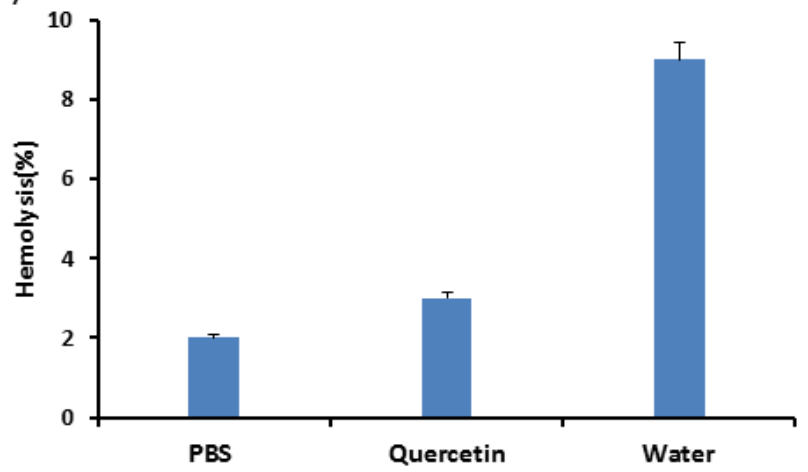

c)

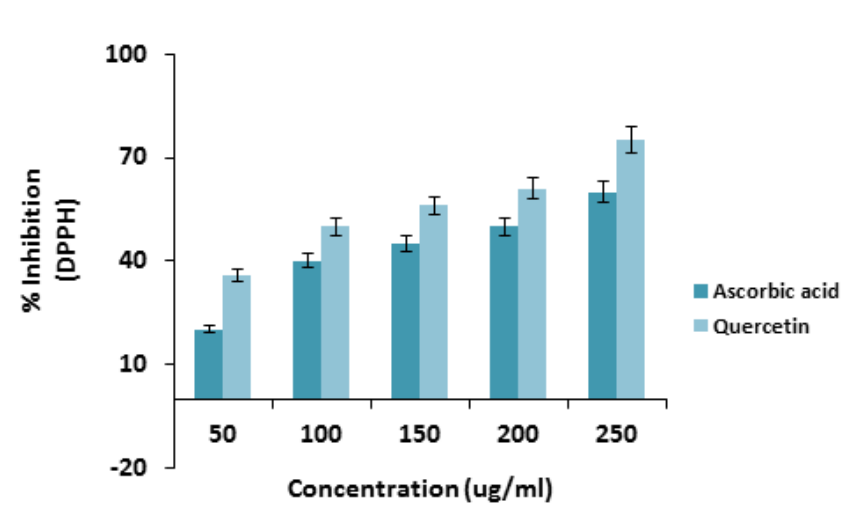

b)

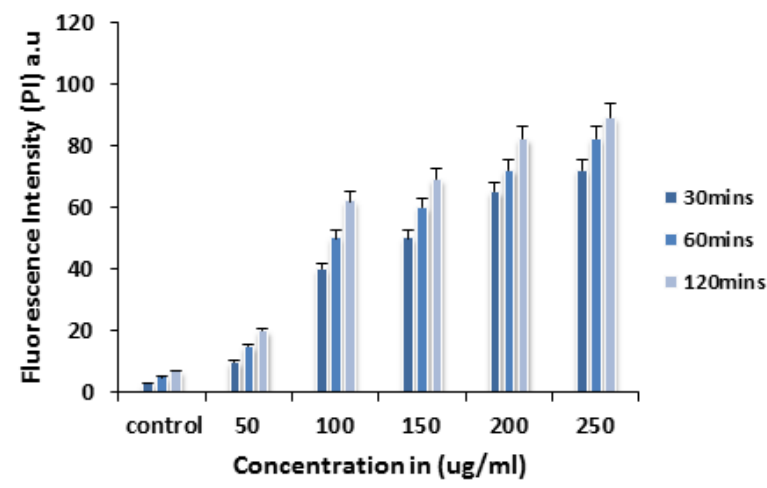

d)

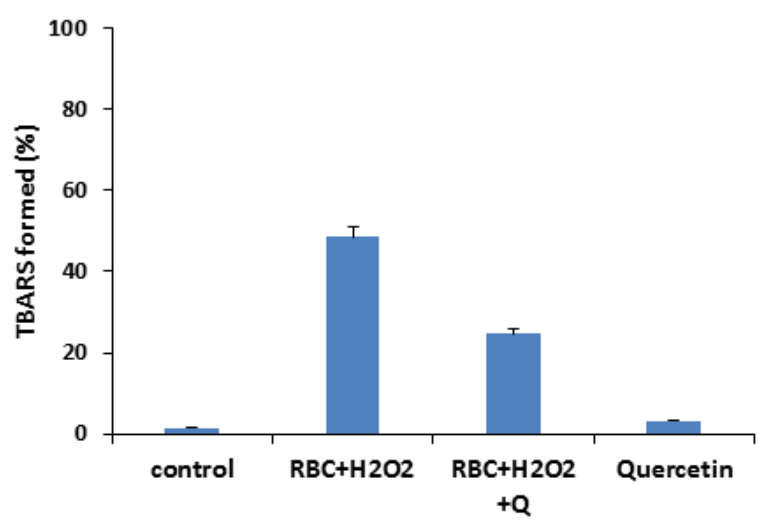

Figure 3. a) Hemolytic activity of bioactive quercetin, with positive and negative control. b) Membrane permeabilization of S.aureus by bioactive quercetin. Fluorescence intensity of PI in S.aureus cells exposed to bioactive quercetin. c) DPPH free radical scavenging of bioactive quercetin at different concentration. d) Percentage thiobarbituric acid substance formed in oxidative assaulted RBC with quercetin and without quercetin. These data represent mean \pm S.D of triplicates. $(n=6)$.

Quercetin is a hydroxylated phenolic substance that belongs to the class of flavonols in which their activities are structure dependent. Several mechanisms of antioxidant activity is influenced by the configuration, substitution, and total number of hydroxyl group in the structure [24]. Flavonoid protects lipids by various mechanisms [25]. In particular, quercetin is able to reduce highly oxidizing free radicals. Quercetin is able to inhibit free radical generation due to its chelating property with metal ions [26]. As a result, the mode of antimicrobial action may be related to their ability to inactivate microbial adhesion, enzymes, and disruption of microbial 
membranes. To further demonstrate the mechanism of quercetin against biofilm, bacteria intracellular reactive oxygen species generation was measured when quercetin was incubated with the bacterial cells and the dye dichlorofluorescein diacetate (DCFH-DA) was used to detect the ROS production. The increased ROS production caused damage to the bimolecular targets like protein, DNA, and lipids with an improper bacterial virulence factor expression ultimately leading to biofilm inhibition. Remarkable increase of ROS level presumably revealed that most cells underwent cellular transformation which makes the cell more vulnerable to exogenous factor [27] causing irreversible cellular damage [28] with membrane distortion or bacterial cell lysis which ultimately leads to cell death. The generation of ROS further damages the biofilm integrity and architecture which is mainly due to the damage in the eDNA, protein, and polysaccharides present in the biofilm matrix.

a)

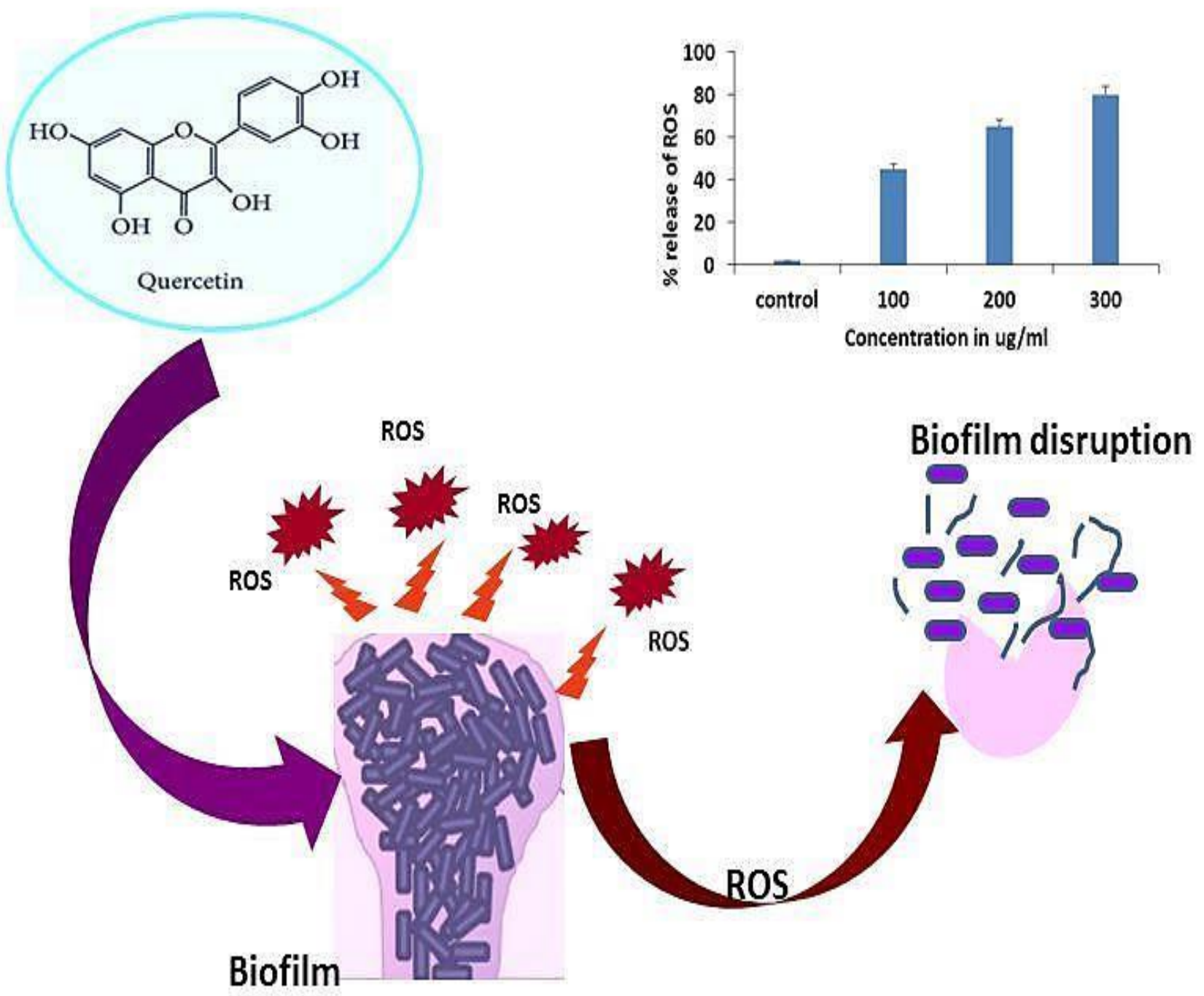

Figure 4. a) Scheme for mechanism of reactive oxygen species (ROS) mediated disruption of biofilm. b) Percentage release of ROS at different concentration of bioactive quercetin. 


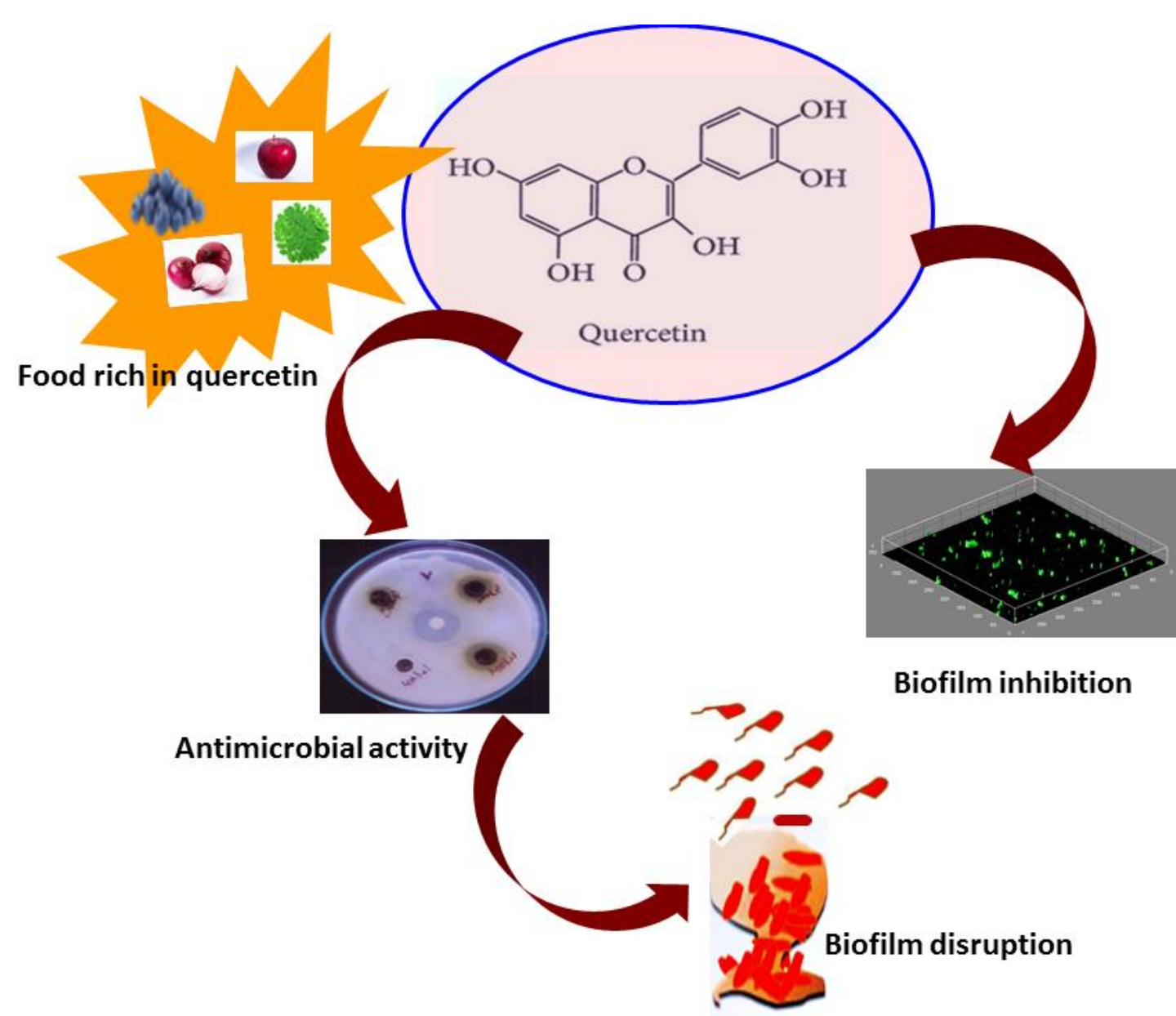

Figure 5. Schematic representation of Quercetin application. Quercetin, which is abundant in many fruits and vegetables, has significant application in combating infectious diseases exhibiting antimicrobial activity and biofilm inhibition and disruption.

Therefore, the results obtained demonstrates (Figure 5) the beneficial role of quercetin in biofilm inhibition, which has significant free radical scavenging activity and membrane activity. These results reiterate the protective role of quercetin in combating human diseases.

\section{CONCLUSION}

This study documents the decisive role of Aspergillus niger in the production of quercetin enhancing the yield. Our study demonstrates the replacement of the chemical process with improved enzyme performance for the synthesis. Significantly, the quercetin produced by microbial transformation demonstrates antimicrobial activity against S.aureus by effectively inhibiting the growth and dispersion of preformed biofilms. Furthermore, the intracellular release of ROS emphasizes its interaction with bacterial biofilm, causing bacterial cell death and finally disruption. As a result, quercetin exerts its action on bacterial biofilms through ROS mediated 
mechanism leading to eradication of biofilms. Moreover, expanding the scope of quercetin towards clinical relevance would provide further insight into the therapeutic strategy.

List of Abbreviations: ROS, Reactive oxygen species; PBS, phosphate buffered saline; HPTLC, High performance thin layer liquid chromatography; DPPH, 1, 1-Diphenyl-2-picryl-hydrazyl; DCFH-DA, dichloro-dihydro-fluorescein diacetate; ANOVA, Analysis of variance; LPO, Lipid peroxidation.

Authors' Contributions: S.S. contributed to the design of the study. S.S and A.J conducted the study and analysis. S.S wrote the manuscript. All authors contributed to the proof reading of the manuscript.

Competing Interests: The authors declare that they have no conflict of interest.

Acknowledgement and Funding: I would like to thank Dr. Smith from biosym for providing the RBC sample. We would like to thank our colleagues for helping us through this project. We received no other funding.

\section{REFERENCES}

1. Moon YJ, Wang X, Morris ME: Dietary flavonoids: effects on xenobiotic and carcinogen metabolism. Toxicol in Vitro 2006, 20 (2):187-210.

2. Perron NR, Brumagin JL: A review of the antioxidant mechanisms of polyphenol compounds related to iron binding. Cell Biochem Biophys 2009, 53:75-100.

3. Parsek MR, Singh PK: Bacterial biofilms: an emerging link to disease pathogenesis. Annu Rev Microbial 2003, 57: 677-701.

4. Simoes M, Bennett RN, Rosa EA: Understanding antimicrobial activities of phytochemicals against multidrug resistant bacteria and biofilms. Natural product reports 2009, 2746-757.

5. Landini P, Antoniani D, Burgess JG, Nijland R: Molecular mechanisms of compounds affecting bacterial biofilm formation and dispersal. Applied Microbiology and Biotechnology 2009, 8: 813-823.

6. Krishnamurty HG, Simpson FJ: Degradation of rutin by Aspergilis flavus. The Journal of Biological Chemistry 1970, 245(6): 1467-1471.

7. Mataraci E, Dosler S: In vitro Activities of antibiotics and antimicrobial cationic peptides alone and in combination against methicillin-resistant Staphylococcus aureus biofilms. Antimicrob Agents Chemother 2012, 56: 6366-6371.

8. Lee LH, Cho MH, Lee J: 3-Indolylacetonitrile decreases Escherichia coli O157:H7 biofilm formation and Pseudomonas aeruginosa virulence. Environ Microbiol 2011, 13: $62-73$. 
9. Konai MM, Ghosh C, Yarlagadda V, Samaddar S, Haldar J: Membrane active phenylalanine conjugated lipophilic norspermidine derivatives with selective antibacterial activity. J Med Chem 2014, 57:9409-9423.

10. Yadav MK, Chae SW, Im GJ, Chung JW, Song JJ: Eugenol: a phyto-compound effective against methicillin-resistant and methicillin-sensitive Staphylococcus aureus clinical strain biofilms. PLoS One 2015, 10: e0119564.

11. Yarlagadda V, Akkapeddi P, Manjunath GB, Haldar J: Membrane active vancomycin analogues: a strategy to combat bacterial resistance. J Med Chem 2014, 57: 4558-4568.

12. Ren Z, Chen G, Wei Z, Sang L, Qi M: Hemocompatibility evaluation of polyurethane film with surface-grafted poly (ethylene glycol) and carboxymethyl-chitosan Journal of Applied Polymer Science 2013, 127: 308-315.

13. Niu Y, Ke D, Yang Q, Wang X, Chen Z, An X, Shen X: Temperature-dependent Stability and DPPH Scavenging Activity of Liposomal Curcumin at pH 7.0. Food Chemistry 2012, 135:1377-1382

14. Forkink M, Smeitink JM, Brock R, Willems PM, Koopman WH: Detection and manipulation of mitochondrial reactive oxygen species in mammalian cells. Biochim Biophys Acta 2010, 1797: 1034-1044.

15. Sreelatha S, Padma PR: Antioxidant activity and total phenolic content of Moringa oleifera leaves in two stages of maturity. Plant Foods Hum Nutr 2009, 64:303-311

16. Nam H, Hong S, Shin K, Oh D: Quercetin production from rutin by a thermostable betarutinosidase from Pyrococcus furiosus. Biotechnol Lett 2012; 34: 483-489.

17. Alarcon J, Alderete J, Escobar C, Araya R, Cespedes CL: Aspergillus niger catalyzes the synthesis of flavonoids from chalcones. Biocat Biotransform 2013, 31:160-7.

18. Stalikas CD: Phenolic acids and flavonoids: occurrence and analytical methods. Methods Mol Biol 2010, 610: 65-90.

19. Borges A, Saavedra MJ, Simoes M: The activity of ferulic and gallic acids in biofilm prevention and control of pathogenic bacteria. Biofouling 2012; 28:755-767.

20. Jagani S, Chelikani R, Kim DS: Effects of phenol and natural phenolic compounds on biofilm formation by Pseudomonas aeruginosa. Biofouling 2009, 25(4):321-324

21. Jayaraman P, Sakharkar MK, Lim CS, Tang TH, Sakharkar KR: Activity and interactions of antibiotic and phytochemical combinations against Pseudomonas aeruginosa in vitro. Inter J of Bio Sci 2009. 6: 556-568.

22. Khushnud T, Mousa SA: Potential role of naturally derived polyphenols and their nanotechnology delivery in cancer. Mol Biotechnol 2013, 55:78-86.

23. Prasaana V, Sreelatha S: Synergistic effect of Moringa oleifera attenuates oxidative stress induced apoptosis in Saccharomyces cerevisiae cells. Evidence for anticancer potential. Int J Pharm Bio Sci 2014: 5: 167-177.

24. Radha S, Sreelatha S: Potential bioactive compounds in the flower extracts of Moringa oleifera and its therapeutic properties. Evidence for antioxidant and nutritional intervention. Indo American Journal of Pharmaceutical Research 2016, 6 (11): 71197128. 
25. Furiga A, Lonvaud-Funel A, Dorignac G, Badet C: In vitro anti-bacterial and antiadherence effects of natural polyphenolic compounds on oral bacteria. Journal of App Micr 2008, 105:1470-1476.

26. Heijnen CG, Haenen GR, Oostveen RM, Stalpers EM, Bast A: Protection of flavonoids against lipid peroxidation: The structure activity relationship revisited. Free Radic Res 2002, 36: 575-581.

27. Nishimuro H, Ohnishi H, Sato M, Ohnishi-Kameyama M, Matsunaga I, Naito S, et al.: Estimated daily intake and seasonal food sources of quercetin in Japan. Nutrients 2015, 7: $2345-2358$

28. Zaharoff DA, Rogers CJ, Hance KW, Schlom J, Greiner JW: Chitosan solution enhances both humoral and cell-mediated immune responses to subcutaneous vaccination. Vaccine 2007, 25: 2085-2094. 